\title{
Localization of passive UHF RFID Labels with Kalman Filter
}

\author{
T. Nick and J. Götze \\ Information Processing Lab, TU Dortmund University, Otto-Hahn-Str. 4, 44227 Dortmund, Germany \\ Correspondence to: T. Nick (theresa.nick@tu-dortmund.de)
}

\begin{abstract}
Localization via Radio Frequency Identification (RFID) is frequently used in different applications nowadays. It has the advantage that next to its ostensible purpose of identifying objects via their unique IDs it can simultaneously be used for the localization of these objects. In this work it is shown how Received Signal Strength Indicator (RSSI) measurements at different antennae of a passive UHF RFID label can be combined for localization. The localization is only done based on the RSSI measurements and a Kalman Filter (KF). Because of non-linearities in the measurement function it is necessary to incorporate an Extended Kalman Filter (EKF) or an Unscented Kalman Filter (UKF) where simulations have shown that the UKF performs better than the EKF. Additionally to the selection of the filter there are different possibilities to increase the localization accuracy of the UKF: The advantages of using Reference Tags (RT) or more than one tag per trolley (relative positioning) in combination with an Unscented Kalman Filter are discussed and simulations results show that the localization error can be decreased significantly via these methods. Another possibility to increase the localization accuracy and in addition to achieve a more realistic simulation is the consideration of the angle between reader antenna and tag. Simulation results with the incorporation of different numbers of fixed antennae lead to the conclusion that this is a useful surplus in the localization.
\end{abstract}

\section{Introduction}

Radio Frequency Identification (RFID) Finkenzeller (2008) has become very popular in the last decades. Especially in the field of logistics many different use cases can be found for the identification as well as tracking of objects and goods Raza et al. (1999). A surplus of RFID is the possibility to gain knowledge about the object's identity as well as its position.
In this paper different approaches for the localization of a passive UHF RFID label (LT) with different types of Kalman Filters are shown. Next to the Extended Kalman Filter (EKF) and the Unscented Kalman Filter (UKF) additional information like relative positioning and the usage of Reference Tags (RTs) is used to improve the localization result.

A use case for the such a localization via RFID is the localization of trolleys coming inside and moving outside of a mail distribution center of Deutsche Post AG. With the help of the localization process a database can always track which trolley has been where at which point of time. This allows for missing trolleys to be found easier because possible actual locations are constrained. The problem of the localization of the trolleys though is that there is not only one trolley coming into the mail distribution center or leaving it, but that there are in most cases also trolleys parked on the side of the gates whose tags send their ID back. Those trolleys should not be detected as coming in or leaving because they are stationary. Figure 1 shows such a scenario where a trolley is just about ready to be moved out of the mail distribution center through the left gate and passing through the RFID localization area while many other trolleys are standing on both sides of that gate. In the front part of the picture it can be seen that there is also a trolley which is passing by the RFID area inside the mail distribution center. It is important to be able to distinguish between these trolleys and the ones coming in or going out because in the database the status of the trolleys remaining inside the mail distribution center is not changed. Through the usage of this RFID-based localization the number of missing trolleys should be reduced and therefore the necessity to replace them. Because there are about 80 mail distribution centers nationwide with about 30 gates each the hardware should be as inexpensive as possible.

Compared to existing localization techniques for RFID labels like "LANDMARC" Ni et al. (2004) or its expansion to work with passive tags and in 3-D Khan and Antiwal (2009) this paper's approach is aimed to achieve higher localization 


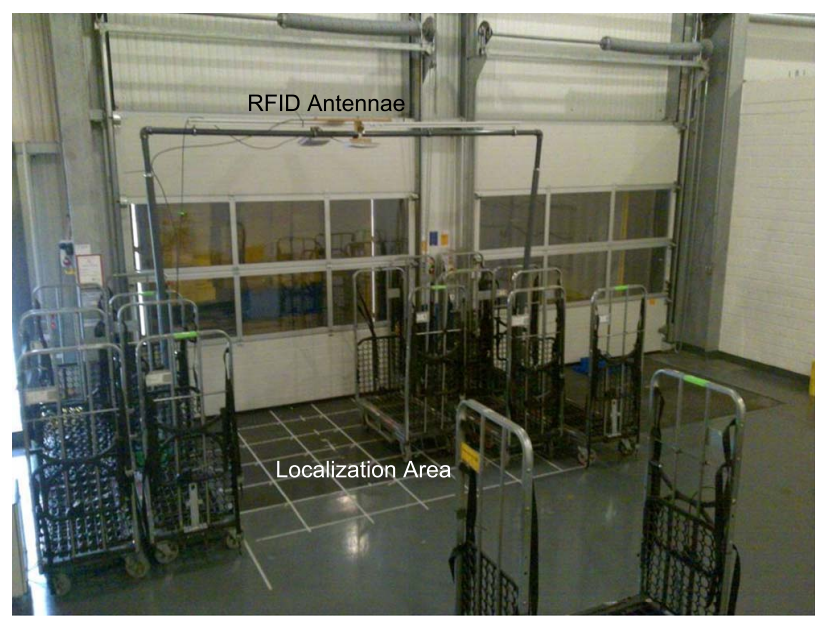

Fig. 1. Test environment for RFID based trolley tracking.

accuracy. This aim should be reached with off-the-shelf hardware components for labels, reader and antennae. That is why a Kalman Filter is chosen which has proven to be well working for such an application Lee et al. (2010). The parameter which is available from almost all RFID readers is the Received Signal Strength Indicator (RSSI). But previous research has shown that RSSI measurements are noisy due to multiple effects of the wireless transmission (like multipath propagation caused by reflections) Parameswaran et al. (2009); Elnahrawy et al. (2004). Therefore the combination of RSSI measurements with a Kalman Filter and additional information might lead to a more accurate localization result.

The paper is organized as follows: In Sec. 2 the mathematical basics about the Extended and Unscented Kalman Filter are explained. In the next section the approaches of differential and relative positioning are shown. Another option is to incorporate the angle between reader antenna and tag into the localization process which is described in Sect. 4. After that the Matlab simulation is explained and its results are given in Sect. 5. The paper closes by giving a conclusion and an outlook on future work in Sect. 6.

\section{Extended and unscented Kalman Filter}

In general a Kalman Filter uses a prediction and an update step to eliminate errors. Because of its structure only the result of the last step and a new measurement are needed to determine a function that describes how the old result is connected to the new one and how the measurement is connected with the prediction result. For a more detailed explanation of Kalman Filters see Thrun et al. (2006).

\subsection{Extended Kalman Filter}

The Extended Kalman Filter uses partial derivatives to be able to cope with non-linearities in the process or measure- ment function. Therefore it is necessary to calculate the Jacobian Matrices. The input into the filter is a Gaussian position estimate of the previous time step $t-1$ with mean $\boldsymbol{\mu}_{t-1}$ and its covariance $\mathbf{P}_{t-1}$ as well as the control $\mathbf{u}_{t}$ and the measurement $\mathbf{z}_{t}$ for updating the position.

In a first step a prediction of the new mean $\overline{\boldsymbol{\mu}}_{t}$ is given by

$\overline{\boldsymbol{\mu}}_{t}=g\left(\mathbf{u}_{t}, \boldsymbol{\mu}_{t-1}\right)$,

where $g()$ represents the relation between the control of the present time step and the mean of the previous time step.

Next is the calculation of a prediction of the new covariance $\overline{\mathbf{P}}_{t}$

$\overline{\mathbf{P}}_{t}=\mathbf{G}_{t} \mathbf{P}_{t-1} \mathbf{G}_{t}^{T}+\mathbf{R}$

where $\mathbf{G}_{t}$ is the Jacobian matrix of the control function and $\mathbf{R}$ is the covariance matrix of the process noise.

With the help of this new covariance the Kalman Gain $\mathbf{K}_{t}$ can be calculated as

$\mathbf{K}_{t}=\overline{\mathbf{P}}_{t} \mathbf{H}_{t}^{T}\left(\mathbf{H}_{t} \overline{\mathbf{P}}_{t} \mathbf{H}_{t}^{T}+\mathbf{Q}\right)^{-1}$

with $\mathbf{H}_{t}$ being the Jacobian matrix of the measurement function and $\mathbf{Q}$ being the covariance matrix of the measurement noise.

Now the new values for the mean $\boldsymbol{\mu}_{t}$ and covariance $\mathbf{P}_{t}$ are computed as

$\boldsymbol{\mu}_{t}=\overline{\boldsymbol{\mu}}_{t}+\mathbf{K}_{t}\left(\mathbf{z}_{t}-h\left(\overline{\boldsymbol{\mu}}_{t}\right)\right)$

and

$\mathbf{P}_{t}=\left(\mathbf{I}-\mathbf{K}_{t} \mathbf{H}_{t}\right) \overline{\mathbf{P}}_{t}$,

where $h()$ is the measurement prediction function. $\mathbf{x}_{t}$ and $\mathbf{P}_{t}$ are the outputs of the Extended Kalman Filter.

\subsection{Unscented Kalman Filter}

The Unscented Kalman Filter Wan and Merwe (2000) can also handle non-linearities in the filtering process. This is done trough stochastic linearization. So-called Sigma Points are used which are located at the mean and symmetrically along the axis of the covariance with two points for each dimension $n$ leading to an overall number of $2 n+1$ Sigma Points.

The input into the Unscented Kalman Filter is as well as for the Extended Kalman Filter a Gaussian position estimate of the previous time step $t-1$ with mean $\boldsymbol{\mu}_{t-1}$ and covariance $\mathbf{P}_{t-1}$. Its first step is to calculate the Sigma Points of the previous step as

$\chi_{t-1}=\left(\begin{array}{lll}\boldsymbol{\mu}_{t-1} & \left.\boldsymbol{\mu}_{t-1}+\gamma \sqrt{\mathbf{P}_{t-1}} \quad \boldsymbol{\mu}_{t-1}-\gamma \sqrt{\mathbf{P}_{t-1}}\right)\end{array}\right.$,

where $\gamma=\sqrt{n+\delta}$ and $\delta=\alpha^{2}(n+\kappa)-n$ with $\alpha$ and $\kappa$ as scaling parameters and $n$ as the dimension of the state space. 
Next these points are propagated through the control function $g$

$\bar{\chi}_{t}^{*}=g\left(\mathbf{u}_{t}, \chi_{t-1}\right)$

and a predicted mean $\overline{\boldsymbol{\mu}}_{t}$ and covariance $\overline{\mathbf{P}}_{t}$ are calculated as

$\overline{\boldsymbol{\mu}}_{t}=\sum_{i=0}^{2 n} w_{m}^{[i]} \bar{\chi}_{t}^{*[i]}$

and

$\overline{\mathbf{P}}_{t}=\sum_{i=0}^{2 n} w_{c}^{[i]}\left(\overline{\boldsymbol{\chi}}_{t}^{*[i]}-\overline{\boldsymbol{\mu}}_{t}\right)\left(\overline{\boldsymbol{\chi}}_{t}^{*[i]}-\overline{\boldsymbol{\mu}}_{t}\right)^{T}+\mathbf{R}$,

where $w_{m}$ and $w_{c}$ are the weights according to

$w_{m}^{[0]}=\frac{\delta}{n+\delta}$

$w_{c}^{[0]}=\frac{\delta}{n+\delta}+\left(1-\alpha^{2}+\beta\right)$ and

$w_{m}^{[i]}=w_{c}^{[i]}=\frac{1}{2(n+\delta)} \quad$ for $i=1, \ldots, 2 n$

with $\beta$ as a scaling parameter and $\mathbf{R}$ as the covariance matrix of the process noise.

With the help of $\overline{\boldsymbol{\mu}}_{t}$ and $\overline{\mathbf{P}}_{t}$ new Sigma Points $\bar{\chi}_{t}$ are calculated

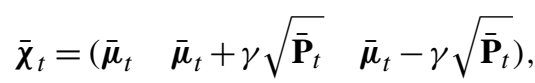

which now capture the uncertainty after the prediction step.

For each of these Sigma Points a predicted observation point is computed through the measurement function $h$

$\overline{\mathbf{Z}}_{t}=h\left(\bar{\chi}_{t}\right)$.

Now $\overline{\mathbf{Z}}_{t}$ is used to calculate the predicted observation $\hat{\mathbf{z}}_{t}$ and its uncertainty $\mathbf{S}_{t}$ according to

$\hat{\mathbf{z}}_{t}=\sum_{i=0}^{2 n} w_{m}^{[i]} \overline{\mathbf{Z}}_{t}^{[i]}$

and

$\mathbf{S}_{t}=\sum_{i=0}^{2 n} w_{c}^{[i]}\left(\overline{\mathbf{Z}}_{t}^{[i]}-\hat{\mathbf{z}}_{t}\right)\left(\overline{\mathbf{Z}}_{t}^{[i]}-\hat{\mathbf{z}}_{t}\right)^{T}+\mathbf{Q}$,

where $\mathbf{Q}$ is the covariance of the additive measurement noise.

For the calculation of the Kalman Gain $\mathbf{K}_{t}$ the cross-covariance $\overline{\mathbf{P}}_{t}^{x, z}$ between state and observation is needed

$\overline{\mathbf{P}}_{t}^{x, z}=\sum_{i=0}^{2 n} w_{c}^{[i]}\left(\overline{\boldsymbol{\chi}}_{t}^{[i]}-\overline{\boldsymbol{\mu}}_{t}\right)\left(\overline{\mathbf{Z}}_{t}^{[i]}-\hat{\mathbf{z}}_{t}\right)^{T}$,

which leads to

$\mathbf{K}_{t}=\overline{\mathbf{P}}_{t}^{x, y} \mathbf{S}_{t}^{-1}$
Now the outputs $\boldsymbol{\mu}_{t}$ and $\mathbf{P}_{t}$ of the Unscented Kalman Filter can be computed with the help of the measurement $\mathbf{z}_{t}$ as

$\boldsymbol{\mu}_{t}=\overline{\boldsymbol{\mu}}_{t}+\mathbf{K}_{t}\left(\mathbf{z}_{t}-\hat{\mathbf{z}}_{t}\right)$

and

$\mathbf{P}_{t}=\overline{\mathbf{P}}_{t}-\mathbf{K}_{t} \mathbf{S}_{t} \mathbf{K}_{t}^{T}$

\section{Differential and relative positioning}

In Nick et al., 2011a,b approaches are shown how to localize a passive UHF RFID label with an Unscented Kalman Filter and the help of relative positioning or reference tags, respectively. The basic principles of both methods are given in the following.

\subsection{Relative positioning}

For the relative positioning the object is not only equipped with one $(N=1)$ UHF RFID tag, but with $N>1$ tags that have a fixed relative position to each other. In most cases it is sufficient to equip the object with $N=2$ tags. This hardly adds cost to the localization method and achieves the highest increase in localization accuracy due to the relative position information between the two labels.

When needed in the measurement function $h$ (see Eq. 11) of the Unscented Kalman Filter the RSSI value is transformed into a distance. With the help of these distances $d_{i}$ from each reader antenna to each tag the $\mathrm{x}-, \mathrm{y}-$ and $\mathrm{z}-$ coordinates of the RFID tags can be computed. For the distances $d_{i}$ holds:

$d_{i}=\sqrt{\left(x_{i}-x\right)^{2}+\left(y_{i}-y\right)^{2}+\left(z_{i}-z\right)^{2}}$,

where $x_{i}, y_{i}$ and $z_{i}$ are the coordinates of the reader antenna $i(i=1, \ldots, \mathrm{M}, \mathrm{M}=$ number of antennae $)$ and $x, y$ and $z$ are the coordinates of the label that has to be located.

When the object that should get localized is equipped with two RFID labels additional information next to the RSSI values can be used for the localization. The two tags always have the same relative position to each other (the same constant distance between each other in all three coordinates). As a second information you know that these two tags need to be moving with the same velocity. These data can be given to the measurement function $h$ of the Kalman Filter and the measurement vector $\mathbf{z}_{t}$ has to be build accordingly to these parameters as well

$\mathbf{z}_{t}=\left[\begin{array}{c}d_{i 1} \\ d_{i 2} \\ d_{i s t_{x}} \\ \text { dist }_{y} \\ \text { dist }_{z} \\ 0 \\ 0 \\ 0\end{array}\right]$. 
The first $2 \cdot M$ components $d_{i 1}$ and $d_{i 2}$ of the vector are the distances calculated from the RSSI measurements. The next three values are the known fixed distances of the two tags and the last three values are the differences in velocities in all three directions which should be zero because the tags are fixed to the same object.

The additional information about the constant distances and equal velocitites transform the UKF into a Constrained Unscented Kalman Filter (CUKF) Simon (2010). The type of CUKF used here is called "Perfect Measurement" because it augments the measurement vector by components which are known.

\subsection{Differential Positioning with Reference Tags (RTs)}

By placing Reference Tags (RTs) with known location in the area in which another tag shall be located more information is available for the localization process. The RTs answer to the reader queries just like every other tag. That way more RSSI values are collected. This information can be used for localizing the tagged object.

RSSI values of the RTs and the $k$ nearest neighbor $(\mathrm{kNN})$ method Duda et al. (2000) are used to achieve a better localization result. For the unknown tag (LT) all RSSI measurements $t_{i}$ to the $M$ antennae are written in vector $\mathbf{t}$

$\mathbf{t}=\left[\begin{array}{ll}t_{1} & t_{2} \ldots t_{M}\end{array}\right]$.

The RSSI values from the $N$ RTs to all $M$ antennae are inserted into matrix $\Theta$

$\boldsymbol{\Theta}=\left[\begin{array}{cccc}\Theta_{11} & \Theta_{12} & \ldots & \Theta_{1 M} \\ \vdots & & & \vdots \\ \Theta_{N 1} & \Theta_{N 2} & \ldots & \Theta_{N M}\end{array}\right]$.

Based on these RSSI values the Euclidean distance between each RT and the LT can be calculated as

$e_{j}=\sqrt{\sum_{i=1}^{M}\left(\Theta_{j i}-t_{i}\right)^{2}}$

where $j \in(1, N)$ and $e_{j}$ is the distance between the $j$ th RT and the LT. For all $N$ RTs this leads to a vector e which contains the distances between the LT and all RTs in descending order.

Based on the measure of the Euclidean distance the $k$ nearest neighbors are chosen. The value of $k$ can be set to best fit to the problem that is solved with the algorithm in different application scenarios. In a next step the weights $w_{i}(i=1, \ldots, k)$ of these $k$ nearest neighbors are calculated according to

$w_{i}=\frac{1 / e_{i}^{2}}{\sum_{i=1}^{k}\left(1 / e_{i}^{2}\right)}$.

This form of computing the weights implies that the nearer a $\mathrm{RT}$ is to the LT the higher its weight is.
If the $\mathrm{kNN}$ algorithm would be used for the localization of the LT its position in $x$-, $y$ - and $z$-coordinates could now be calculated like

$(x, y, z)=\sum_{i=1}^{k} w_{i}\left(x_{R T_{i}}, y_{R T_{i}}, z_{R T_{i}}\right)$

like it is done in $\mathrm{Ni}$ et al. (2004).

However here the kNN method is used as an additional information for the Unscented Kalman Filter. Therefore the kNN result will be handled differently here.

Usually the measurement function $h$ contains the distance information $d_{i}(i=1, \ldots, M)$ from the LT to all $M$ antennae (from Eq. 18) and the matching measurement vector $\mathbf{z}_{t}$ contains the distances based on the measured RSSI values for the LT. Now with additional information through the RTs $h$ also contains $k$-times the actual position estimates in $\mathrm{x}^{-}, \mathrm{y}-$ and $\mathrm{z}$-coordinate before incorporating the measurement.

Those position estimates can be said to be equal to the positions of the RTs plus additive noise which is comprised in the covariance matrix of the measurement noise $\mathbf{Q}$. The inverse of the weights calculated in Eq. (23) are used here. This form of choosing the noise for the lower part of the matrix leads to a higher noise when the weight is smaller which is due to the fact that the RT is further away from the LT.

To fit to the measurement function $h$ the measurement vector $\mathbf{z}_{t}$ consists of two parts as well. The first part of the vector comprises the distance measurements $d_{i}(i \in(1, M))$ based on the RSSI values from the LT to all $M$ antennae. The rest of the vector contains the $\mathrm{x}-, \mathrm{y}$ - and $\mathrm{z}$-coordinates of those RTs which are the $k$ nearest to the LT.

\section{Angle-dependent RSSI measurements}

As described in Nick et al. (2011c) the angle between reader antenna and tag has an influence on the measured RSSI value. Therefore it is useful to take this angle into consideration when localizing an object which is equipped with an RFID label.

Based on the RSSI measurement in the directivity of the antenna a distance $D$ between antenna and tag can be calculated. With this distance $D$, the position of the antenna $O$ and the directivity a circle can be set up as can be seen in Fig. 2. On every point of this circle with radius $r=D / 2$ the same RSSI value is measured. Based on the radius $r_{i}$ of every of those circles for each antenna the localization can be done via the UKF.

The description via circles is only possible in this case because it reflects the antenna's properties. If an antenna with a differently formed beam is used it is necessary to employ another geometric form. For example it might be suitable for some antennae to use an ellipse instead of the circle to describe the points where the same RSSI value is measured.

The measurement function $h$ of the Unscented Kalman Filter only contains the distance measurements in this case. But 


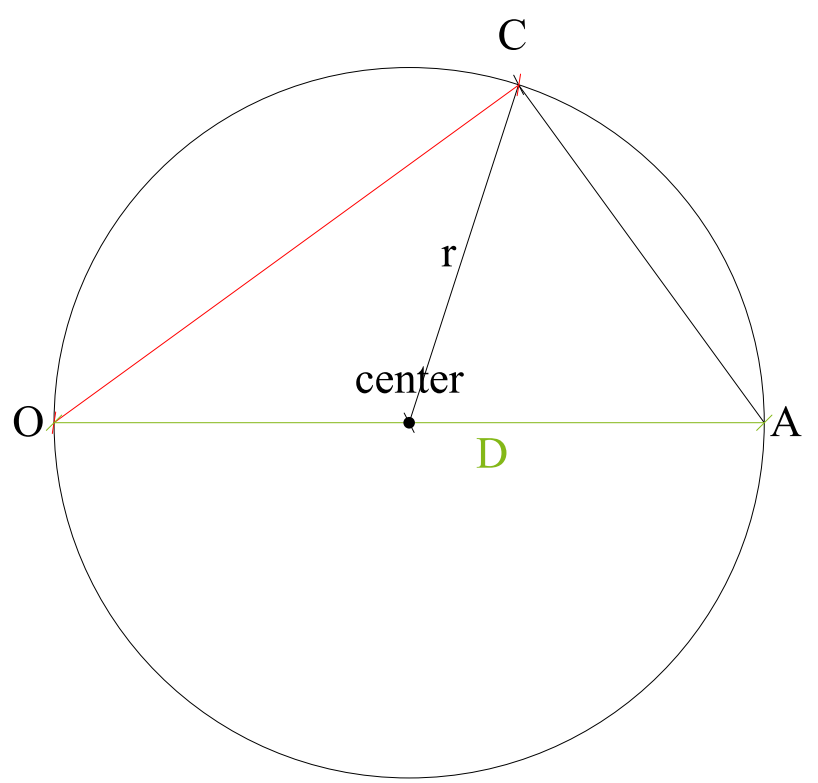

Fig. 2. Method with circle.

compared to the relative or differential methods, in this case the distance is not calculated between reader antenna and tag. For the angle-dependent approach the distance between the center of the circle and the tag is calculated based on the computed radii $r_{i}$ from all $i=1, \ldots, M$ antennae used.

\section{Simulations}

The simulation tool of the 3D localization of passive UHF RFID labels is written in Matlab. The considered space is $5 \mathrm{~m} \times 5 \mathrm{~m} \times 5 \mathrm{~m}$ in which the antennae (and the RTs) are placed and the LT is moving For the movement of the tag some way points are given between which other points are interpolated based on the time interval $d t$ and the velocity $v$. At every way point the RSSI values to all used antennae are calculated. For simulating more realistic scenarios noise is added to these computed RSSI values to take into account disturbances in real world scenarios. These calculated noisy RSSI values are then used as measurement inputs for the Kalman Filter.

The result of the simulation shows the localization error of the Kalman Filter over the desired path. It is the Root Mean Square Error (RMSE) over the estimates of the filter used for the localization. The first 10 results are left out since the filter needs an adjustment period which will take about this number of position estimates (depends on the chosen initial values).

For all methods described in the previous sections the simulation of the localization is carried out with a different number of antennae. The results of the simulations can be seen in Table 1 for the Extended and the Unscented Kalman Filter. Table 2 shows the results for the relative positioning
Table 1. Results of 3-D localization for EKF and UKF.

\begin{tabular}{ccc}
\hline $\begin{array}{r}\text { \# of } \\
\text { Ant }\end{array}$ & Error EKF & Error UKF \\
\hline 2 & $86.18 \mathrm{~cm}$ & $69.06 \mathrm{~cm}$ \\
3 & $66.75 \mathrm{~cm}$ & $49.30 \mathrm{~cm}$ \\
4 & $43.45 \mathrm{~cm}$ & $27.18 \mathrm{~cm}$ \\
\hline
\end{tabular}

Table 2. Results of 3-D localization of UKF_RP and UKF_RT.

\begin{tabular}{ccc}
\hline $\begin{array}{l}\text { \# of } \\
\text { Ant }\end{array}$ & Error UKF_RP & Error UKF_RT \\
\hline 2 & $65.84 \mathrm{~cm}$ & $68.41 \mathrm{~cm} \mathrm{(3)}$ \\
3 & $39.81 \mathrm{~cm}$ & $45.90 \mathrm{~cm} \mathrm{(2)}$ \\
4 & $25.60 \mathrm{~cm}$ & $26.82 \mathrm{~cm} \mathrm{(2)}$ \\
\hline
\end{tabular}

(UKF_RP) as well as the differential positioning (UKF_RT). The number in brackets behind the localization results of the UKF_RT denotes the number of reference tags used for the $k N N$ positioning. The reference tags are placed with a distance of $1 \mathrm{~m}$ from each other in all three dimensions with a total of 36 RTs placed in the considered localization area.

It becomes obvious that the Extended Kalman Filter is outperformed by the UKF for all different number of antennae used. When incorporating additional information into the localization process the UKF_RP achieves better results than the UKF_RT, but both approaches are able to improve the localization accuracy compared to the UKF. Figure 3 shows the results of the different methods for an exemplary path. The colored markers denote the positions of the reader antennae of which three are used for this example.

These simulations have not taken the angle between reader antenna and tag into account so far. When considering the angle for the localization with an Unscented Kalman Filter (UKF_wa) the results as shown in Table 3 are achieved.

The results of the UKF_wa have almost the same localization error compared to the UKF_RT which performed best so far. This leads to the conclusion that the incorporation of the angle is important for a good localization result and it makes the simulation more realistic as well because it reflects the antenna's properties. An important parameter to achieve these good localization results with the UKF_wa is the directivity of the antenna. That means it is of importance to choose wisely how each of the antennae used is aligned according to the path on which the tagged object is moving. Slight changes in the alignment may result in huge variations of the localization error. 


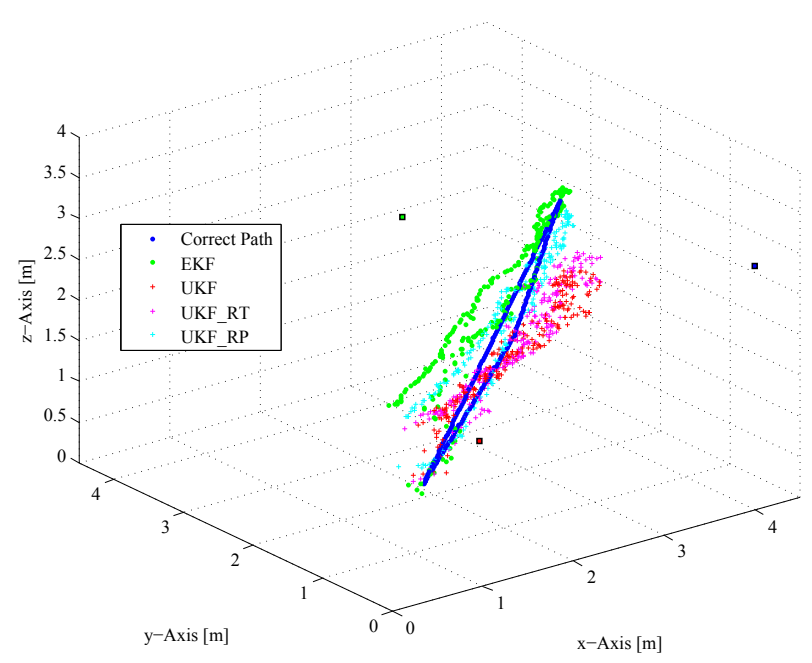

Fig. 3. Localization results of different Kalman Filter with 3 antennae.

Table 3. Results of 3-D localization of UKF_wa.

\begin{tabular}{cc}
\hline $\begin{array}{c}\text { \# of } \\
\text { Ant }\end{array}$ & Error UKF_wa \\
\hline 2 & $65.96 \mathrm{~cm}$ \\
3 & $38.79 \mathrm{~cm}$ \\
4 & $26.61 \mathrm{~cm}$ \\
\hline
\end{tabular}

\section{Conclusion and future work}

In this paper it is shown through simulations that it is possible to localize a passive UHF RFID label solely with RSSI measurements and additional information combined in a Kalman Filter. The localization results of the Unscented Kalman Filter are better than these of the Extended Kalman Filter. The accuracy of the localization result can further be improved by the usage of reference tags or an additional tag on the object. When incorporating the angles between reader antennae and the tag into the localization process the simulation becomes more realistic and an accurate localization is possible as well.

For the future these simulation results will be verified through real world measurements. The results gained from these measurements might differ from the simulation results due to multipath propagations in the localization environment. Therefore it can be useful to incorporate a camera into the localization process which is independent of these effects. To be able to view the results of the RFID as well as the camera-assisted localization easily it might be useful to implement a demonstration tool to show the different localization results.
Acknowledgements. The reported $\mathrm{R}+\mathrm{D}$ work was carried out in the frame of the BMBF-Project smaRTI (Smart ReUsable Transport Items); the smaRTI project is carried out in the frame of the Efficiency Cluster Logistic Ruhr (part of the Leading-Edge Cluster - High-Tech Strategy for Germany). This particular research was supported by the BMBF (Bundesministerium fuer Bildung und Forschung) of Federal Republic of Germany under grant 01IC10L10H (Sichere, flexible Detektion und Lokalisierung von UHF RFID-Labeln in rauer Umgebung). The responsibility for this publication is held by the authors only. In particular we have to thank our industrial partners Infineon Technologies AG and Deutsche Post AG supporting us by the required information regarding the RFID test environment and tag data.

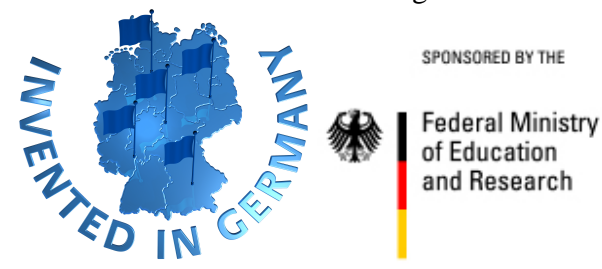

\section{References}

Duda, R., Hart, P., and Stork, D.: Pattern Classification, WileyInterscience, 174-191, 2000.

Elnahrawy, E., Li, X., and Martin, R.: The Limits of Localization Using Signal Strength: A Comparative Study, in: Proceedings of IEEE Sensor and AdHoc Communications and Networks (SECON), 2004.

Finkenzeller, K.: RFID Handbuch, Hanser Fachbuchverlag, 5th edn., 2008.

Khan, M. and Antiwal, V.: Location Estimation Technique using Extended 3-D LANDMARC Algorithm for Passive RFID Tag, in: Proceedings of 2009 IEEE International Advance Computing Converence (IACC 2009), 2009.

Lee, K.-C., Oka, A., Pollakis, E., and Lampe, L.: A Comparison between Unscented Kalman Filtering and Particle Filtering for RSSI-Based Tracking, in: Proceedings of WPNC, 2010.

Ni, L., Liu, Y., Lau, Y., and Patil, A.: LANDMARC: Indoor Location Sensing Using Active RFID, Wireless Networks, 10, 701710, 2004.

Nick, T., Goetze, J., John, W., and Stoenner, G.: Localization of Passive UHF RFID Labels Using An Unscented Kalman Filter with Relative Position Information, in: Proceedings of European Workshop on Smart Objects: Systems, Technologies and Applications (RFID-SysTech 2011), 2011a.

Nick, T., Goetze, J., John, W., and Stoenner, G.: Localization of UHF RFID Labels with Reference Tags and Unscented Kalman Filter, in: Proceedings of IEEE International Conference on RFID-Technologies and Applications (RFID-TA 2011), 2011b.

Nick, T., Khan, M. Z. I., Goetze, J., and John, W.: Localization of Passive UHF RFID Label with Unscented Kalman Filter Based on Angle-Dependent RSSI Measurements, in: Proceedings of IEEE 5th International Conference on Signal Processing and Communication Systems (IEEE ICSPCS), 2011c.

Parameswaran, A., Husain, M., and Upadhyaya, S.: Is RSSI a Reliable Parameter in Sensor Localization Algorithms - An Experimental Study, in: Proceedings of Field Failure Data Analysis Workshop (F2DA’09), 2009. 
Raza, N., Bradshaw, V., and Hague, M.: Applications of RFID Technology, in: Proceedings of IEE Colloquium on RFID Technology, 1999.

Simon, D.: Kalman filering with state constraints: a survey of linear and nonlinear algorithms, IET Control Theory and Applications, 4, no. 8, 1303-1318, 2010.
Thrun, S., Burgard, W., and Fox, D.: Probabilistic Robotics, The MIT Press, 39-71 2006.

Wan, E. A. and Merwe, R. V. D.: The Unscented Kalman filter for nonlinear estimation, in: Proceedings of IEEE 2000 Adaptive Systems for Signal Processing, Communicationa and Control Symposium, 2000. 